\title{
High-Energy Electron Confinement in a Magnetic Cusp Configuration
}

\author{
Jaeyoung Park, ${ }^{1, *}$ Nicholas A. Krall, ${ }^{2}$ Paul E. Sieck, ${ }^{1}$ Dustin T. Offermann, ${ }^{1}$ Michael Skillicorn, ${ }^{1}$ \\ Andrew Sanchez, ${ }^{1}$ Kevin Davis, ${ }^{1}$ Eric Alderson, ${ }^{1}$ and Giovanni Lapenta ${ }^{3}$ \\ ${ }^{1}$ Energy Matter Conversion Corporation (EMC2), 9155 Brown Deer Road, San Diego, \\ California 92121, USA \\ ${ }^{2} 1070$ America Way, Del Mar, California 92104, USA \\ ${ }^{3}$ Center for Mathematical Plasma Astrophysics, University of Leuven, Celestijnenlaan 200B, \\ 3001 Leuven, Belgium
}

(Received 22 October 2014; published 11 June 2015)

\begin{abstract}
We report experimental results validating the concept that plasma confinement is enhanced in a magnetic cusp configuration when $\beta$ (plasma pressure/magnetic field pressure) is of order unity. This enhancement is required for a fusion power reactor based on cusp confinement to be feasible. The magnetic cusp configuration possesses a critical advantage: the plasma is stable to large scale perturbations. However, early work indicated that plasma loss rates in a reactor based on a cusp configuration were too large for net power production. Grad and others theorized that at high $\beta$ a sharp boundary would form between the plasma and the magnetic field, leading to substantially smaller loss rates. While not able to confirm the details of Grad's work, the current experiment does validate, for the first time, the conjecture that confinement is substantially improved at high $\beta$. This represents critical progress toward an understanding of the plasma dynamics in a high- $\beta$ cusp system. We hope that these results will stimulate a renewed interest in the cusp configuration as a fusion confinement candidate. In addition, the enhanced high-energy electron confinement resolves a key impediment to progress of the Polywell fusion concept, which combines a high- $\beta$ cusp configuration with electrostatic fusion for a compact, power-producing nuclear fusion reactor.
\end{abstract}

DOI: 10.1103/PhysRevX.5.021024

\section{BACKGROUND}

The use of magnetic fields to confine high-temperature plasmas has been one of the main pathways pursued in controlled thermonuclear fusion research since the 1950s. Several magnetic field configurations, such as magnetic pinch, stellarator, magnetic mirror, and tokamak, have been explored to achieve net power generation from fusion reactions [1-3]. However, one of the critical technical challenges related to magnetically confined fusion devices is the plasma instability inside the confining magnetic fields. For example, magnetohydrodynamic instabilities driven by plasma current or plasma pressure, such as kink and Rayleigh-Taylor instabilities, can abruptly disrupt the plasma confinement by tearing apart confining magnetic fields and expelling the plasma. Such plasma instabilities limit the maximum operating plasma current or pressure in the device and increase the reactor size required to achieve net fusion power. Moreover, a large engineering safety margin is typically required to prevent reactor failure in the

*jypark@emc2fusion.com

Published by the American Physical Society under the terms of the Creative Commons Attribution 3.0 License. Further distribution of this work must maintain attribution to the author(s) and the published article's title, journal citation, and DOI.
Subject Areas: Plasma Physics

event of a major disruption, increasing engineering complexities and reactor cost.

In comparison, the magnetic cusp configuration provides excellent macroscopic plasma stability due to the convex magnetic field curvature towards the confined plasma system in the center, as shown in Fig. 1(a) [1,2,4]. Experiments on the cusp field configuration have confirmed the stability

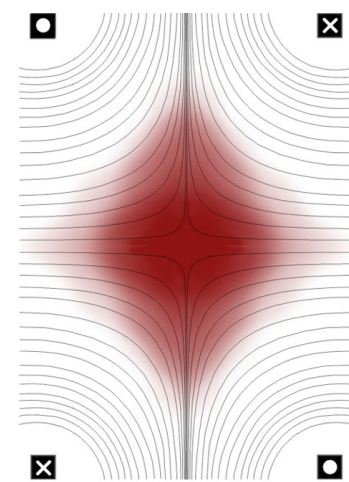

(a)

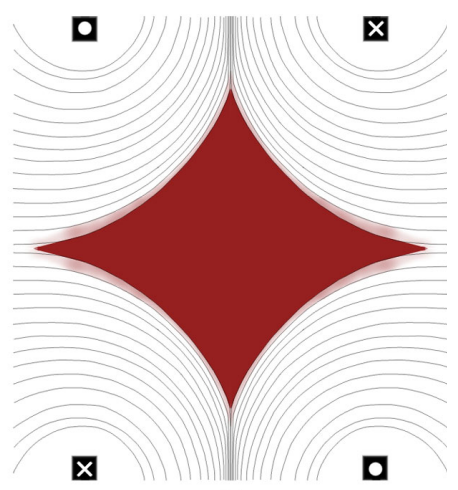

(b)
FIG. 1. Comparison of the predicted magnetic geometry at low $\beta$ and at high $\beta$ in a magnetic cusp configuration, with (a) low $\beta$ and (b) high $\beta$. Note the convex nature of magnetic field lines. The red shading denotes plasma pressure. At high $\beta$ (e.g., $\beta \sim 1$ ), the strong plasma diamagnetism excludes the magnetic fields, which creates a sharp boundary. 
property, even at very high plasma pressures up to $\beta=1$ $[5,6]$. Plasma beta $\beta$ is defined as the ratio of plasma pressure to confining magnetic field pressure, $\beta=P_{\text {plasma }} /\left(B^{2} / 2 \mu_{0}\right)$, where $P_{\text {plasma }}$ is the plasma pressure, $\mu_{0}$ is the magnetic permeability, and $B$ is the magnetic flux density. In a cusp configuration, the local value of $\beta$ varies from zero where the plasma pressure is low, to infinity at the center where there is a finite plasma pressure with a zero magnetic field. When a single value of $\beta$ is given for the entire volume in this paper, it refers to the average plasma pressure inside the cusp system (near the center) normalized to the magnetic field pressure at the center of one of the magnet coils. Since the fusion power output scales as $\beta^{2}$ for a given magnetic field, high- $\beta$ operation is advantageous for a compact economical fusion reactor. In contrast, the design parameter for the International Thermonuclear Experimental Reactor (ITER), a proposed tokamak device to achieve a net fusion power output, is $\beta \approx 0.03$ [7].

Substantial theoretical and experimental efforts have been devoted to investigating the magnetic cusp configuration $[1,3,4]$. Initial results, however, showed poor plasma confinement [1]. This was thought to be related to the open magnetic field structure and rapid mirrorlike plasma loss in a low- $\beta$ cusp. Grad and others predicted theoretically that the plasma confinement properties of the cusp configuration would be greatly enhanced if the magnetic field exhibits a sharp boundary separating the field-free high$\beta$ plasmas and the vacuum region with magnetic fields, as shown in Fig. 1(b) [1,8]. This change in magnetic field structure is driven by plasma diamagnetism, thus proportional to $\beta$. Figure 1(b) shows schematically a cusp magnetic configuration at $\beta=1$. Equation (1) describes the theoretically estimated plasma loss rate for the cusp system in Fig. 1(b) [8]. The physical idea behind Eq. (1) is as follows: At high $\beta$, plasma approaching this sharp transition layer reflects back into the confined volume due to the discontinuity in the magnetic field. Eventually, however, a plasma particle after many reflections will move almost exactly in the direction of the cusp opening and will be lost. Grad conjectured that this loss hole will have a radius equal to the electron gyroradius, as shown in Eq. (1). By contrast, when $\beta$ is small in the cusp, the transition region is the size of the confined volume, and plasma approaching the boundary attaches to field lines and streams out the cusp. This loss rate is related to the plasma loss rate in a magnetic mirror and is much larger than the rate given in Eq. (1) [9].

Equation (1) shows the electron and ion loss rate for a single cusp with high- $\beta$ plasma,

$$
\frac{I_{e, i}}{e}=\frac{\pi}{9} n_{e, i} v_{e, i} \pi\left(r_{e, i}^{\mathrm{gyro}}\right)^{2},
$$

where $I$ is the loss current, $e$ is the electron charge, $n$ is the density, $v$ is the velocity, $r^{\text {gyro }}$ is the local gyroradius at the cusp location, and subscripts $e$ and $i$ denote electron and ion species, respectively.

Though several experiments were constructed to validate this conjecture, two critical issues limited their efforts $[5,6,10-12]$. The first issue was the engineering and technical challenge related to initially forming a high- $\beta$ plasma, where a required initial injection power is on the order of $100 \mathrm{MW}$ or more. The second issue was the theoretical and experimental difficulty in determining the plasma loss rate in a high- $\beta$ plasma state. It was accepted that if the loss rate is determined by the ion gyroradius, it would be unacceptably large for a fusion power reactor. Experiments seemed to indicate that the ion gyroradius did indeed dominate the loss rate [11], though another work suggested that a hybrid radius between electron and ion gyroradius gives the correct loss rate [12]. Because of these problems, the concept of a fusion power reactor based on a cusp magnetic field was largely abandoned, until a new idea, discussed in the next section, was proposed that retains the advantages of cusp confinement but removes the issue of the ion gyroradius dominating the loss rates.

\section{POLYWELL FUSION CONCEPT}

In 1985, Bussard introduced a fusion concept, the "Polywell" reactor, which combines the magnetic cusp configuration with the inertial electrostatic confinement fusion concept [13-15]. In the Polywell reactor, electrons are confined magnetically by a cusp field while ions are confined by an electrostatic potential well produced by electron beam injection. The use of an electron beam provides two critical advantages for the Polywell reactor over other magnetic cusp devices. First, the excess electrons from the beam form an electrostatic potential well [16-18]. By utilizing an electrostatic potential well, the Polywell reactor employs a highly efficient method to accelerate ions to high energies for fusion. Second, the potential well, rather than the magnetic field, confines ions. In a Polywell reactor, the main issues of high-temperature plasma containment and plasma heating are thus reduced to the confinement property of the injected electron beam. In previous experiments, a deep potential well was formed and maintained in a cusp field [9]. But that work was limited by the high loss rates typical of a low- $\beta$ cusp confined plasma. To progress further, it is necessary to show that electron confinement is enhanced at high $\beta$, as conjectured by Grad and others.

\section{CONFINEMENT MEASUREMENT AT HIGH $\beta$}

In this paper, we present the first experimental evidence that confinement of high-energy electrons by a magnetic cusp is enhanced in a high- $\beta$ plasma state. Figure 2 shows the experimental setup based on a hexahedral, or 6-coil, Polywell system. 
Test electron beam at $7 \mathrm{kV}$ (at $1-3 \mathrm{~A}$ )

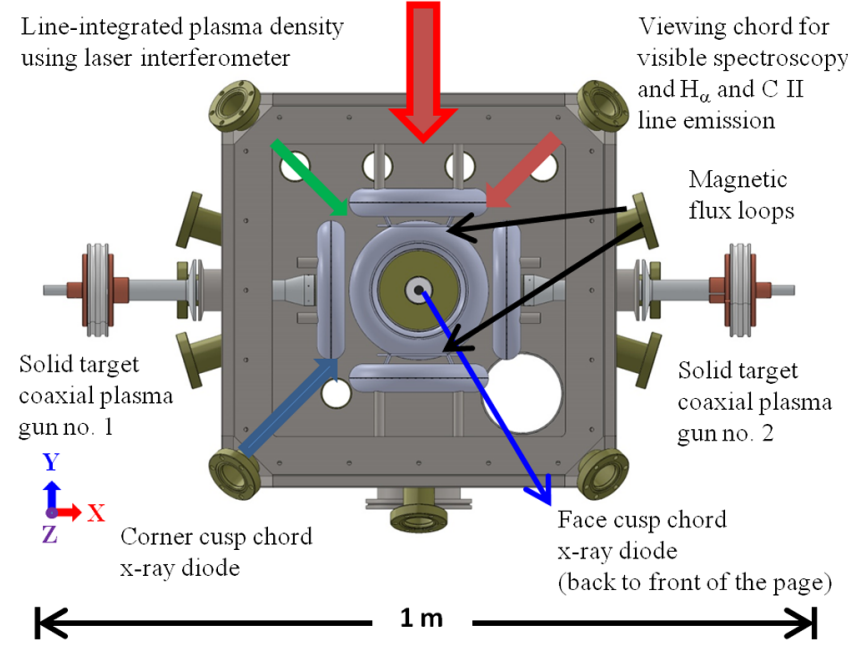

FIG. 2. Experimental setup of high- $\beta$ plasma confinement study using hexahedral cusp configuration.

We conduct the experiments in a cubic vacuum chamber measuring $45 \mathrm{~cm}$ on an edge. Centered in this chamber are six identical magnet coils, each coil having major radius $6.9 \mathrm{~cm}$ and minor radius $1.3 \mathrm{~cm}$. The coils are arranged such that each coil is centered on a face of a cube measuring $21.6 \mathrm{~cm}$ on an edge, as shown in Fig. 2, producing a cusp magnetic field. Each coil is capable of generating a static magnetic field from 0.6 to $2.7 \mathrm{kG}$, as measured at the coil center, corresponding to coil currents between 5-22 kA turns. The coils are energized for pulse duration of $41 \mathrm{~ms}$ and the $L / R$ time of coil is approximately $1 \mathrm{~ms}$. In comparison, the typical plasma duration is $100 \mu \mathrm{s}$; thus, the coils produce static magnetic fields during the time of interest.

The plasma injectors consist of two coaxial plasma guns with an anode to cathode gap spacing of $2 \mathrm{~mm}$ utilizing $\vec{j} \times \vec{B}$ plasma acceleration [10]. The plasma guns use solid polypropylene film with $4 \mu \mathrm{m}$ thickness to generate highpressure plasmas in a very short time compared to more common gas-fed plasma guns. The amount of material injection is controlled by the number of film layers; two layers are chosen for the work presented here. Initial experiments use a single plasma gun and do not produce a sufficiently high $\beta$ in the cusp. The plasma guns are constructed with tungsten electrodes and boron nitride insulators to minimize erosion and impurity injection. Each gun operates with 60-150 kA of gun current using a switched capacitor circuit with an ignitron. Each gun is capable of injecting high-pressure plasma with up to 500-MW pulse power from the capacitor and pulse duration between 5-10 $\mu$ s. This changes $\beta$ from zero to near 1 in 10-20 $\mu \mathrm{s}$, and then $\beta$ decays back to zero. The plasma guns are located $0.5 \mathrm{~cm}$ outside the magnetic cusp points along the $x$ axis.

The electron-beam injector utilizes a Lanthanum hexaboride $\left(\mathrm{LaB}_{6}\right)$ cathode to produce thermionic electron emission into the plasma environment. Beam extraction is obtained with the use of a triple grid system made of graphite grids to provide controlled electron extraction, while limiting plasma bombardment to the cathode surface. The electron-beam injector is located $50 \mathrm{~cm}$ above the top coil along the $y$ axis. Beam currents are produced at 1-3 A with beam energies up to $7 \mathrm{keV}$. The $7-\mathrm{keV}$ electrons have a transit time of $7 \mathrm{~ns}$ in the cusp, allowing time resolved measurements of energetic electron confinement during pulsed plasma injection. The electron beam injection has a limited power $(\sim 20 \mathrm{~kW})$ and so it does not change plasma pressure in the cusp.

The confinement of the 7-keV beam electrons is measured by the hard-x-ray emission from the induced bremsstrahlung inside the cusp system. The x-ray detection system consists of a biased photodiode in a differentially pumped housing, a Kapton black window, a plastic collimator, and a magnetic yoke. The Kapton window is $25 \mu \mathrm{m}$ thick and completely opaque to visible light as well as $\mathrm{x}$ rays below $2 \mathrm{keV}$. The collimator is designed to maximize the core plasma volume in the line of sight for the photodiode, while preventing the detection of $x$ rays produced when beam electrons are incident on the coil surfaces. A magnetic yoke with $2 \mathrm{kG}$ magnetic field is used near the entrance of the collimator to prevent the beamelectron-induced fluorescence of the Kapton window. Two viewing chords are used: one viewing the central confined plasma through the cusp openings in the middle of coils along the $z$ axis, and the other viewing the plasma through the cusp openings among the corner of coils along the $x=y=z$ line. The former view has a collection volume of $620 \mathrm{~cm}^{3}$ and the latter view has a collection volume of $380 \mathrm{~cm}^{3}$.

In the current study, several diagnostics are utilized to measure the plasma $\beta$ in the cusp. A heterodyne laser interferometer is used to measure a line-integrated plasma density through the corner cusp using a green laser at $532 \mathrm{~nm}$. To report line-averaged density values, the plasma length is assumed to be $22 \mathrm{~cm}$, equal to the cusp system diameter. At that length, an average electron density of $1 \times 10^{16} \mathrm{~cm}^{-3}$ is equal to a phase shift of $180 \mathrm{deg}$. An Acton monochrometer with selectable gratings $(2400,600$, and 300 grooves $/ \mathrm{mm}$ ) is used in conjunction with a Princeton Instruments PI-Max intensified CCD camera to acquire time-resolved spectroscopic data for Stark broadening and plasma chemical composition. Two optical fibers collect plasma photoemission from the corner of the Polywell to photodiode detectors. Narrow band interference filters are used on each detector, one for the $H_{\alpha}$ line and the other for the $\mathrm{C}$ II line at $724 \mathrm{~nm}$. In addition, two magnetic flux loops are installed to monitor the plasma diamagnetism. Each loop consists of a single circular turn of a coaxial cable with major radius $5.2 \mathrm{~cm}$ and minor radius $1.1 \mathrm{~mm}$. The loops are located $1 \mathrm{~cm}$ away from the magnet casing surface, such that the plane of each loop is $8.0 \mathrm{~cm}$ from the chamber center. 
To summarize the experimental operation, the plasma guns produce the high- $\beta$ condition and the electron beam is used as a diagnostic to measure electron confinement. The confinement of the injected electron beam is measured by the x-ray diodes via electron-beam-induced bremsstrahlung. Improved confinement should lead to a higher density of high-energy beam electrons, which would give a stronger $x$-ray signal.

Figure 3 shows a time-integrated visible image of a high$\beta$ plasma in the cusp system. The magnetic cusp structure is visible in the plasma, as well as significant plasma nonuniformity in the central region with zero magnetic fields. The light-collection volumes of the $\mathrm{x}$-ray diagnostics are increased to 380 and $620 \mathrm{~cm}^{3}$ to reduce spurious signals from this spatial variation by volume averaging. The central plasma volume visible through the $6.3-\mathrm{cm}$-radius bore of the front-facing coil is $1050 \mathrm{~cm}^{3}$, and the estimated confined plasma volume inside the cusp configuration is $5000 \mathrm{~cm}^{3}$, assuming spheres with respective radii of 6.3 and $10.8 \mathrm{~cm}$. Though additional plasma guns might produce a more uniform plasma, the experimental results with two plasma guns are sufficient to clearly demonstrate the enhanced high-energy electron confinement as shown below. Additional plasma guns will be utilized to investigate the impact of plasma symmetry on confinement in future work.

Figure 4 shows time-resolved experimental results from the various diagnostics from a high- $\beta$ shot, no. 15640 . The coils are energized $40 \mathrm{~ms}$ prior to plasma injection and the coil currents are kept at a constant value during the time period shown in Fig. 4, with the vacuum $B$-field value of $2.7 \mathrm{kG}$ at the cusp points near the plasma guns. In addition,

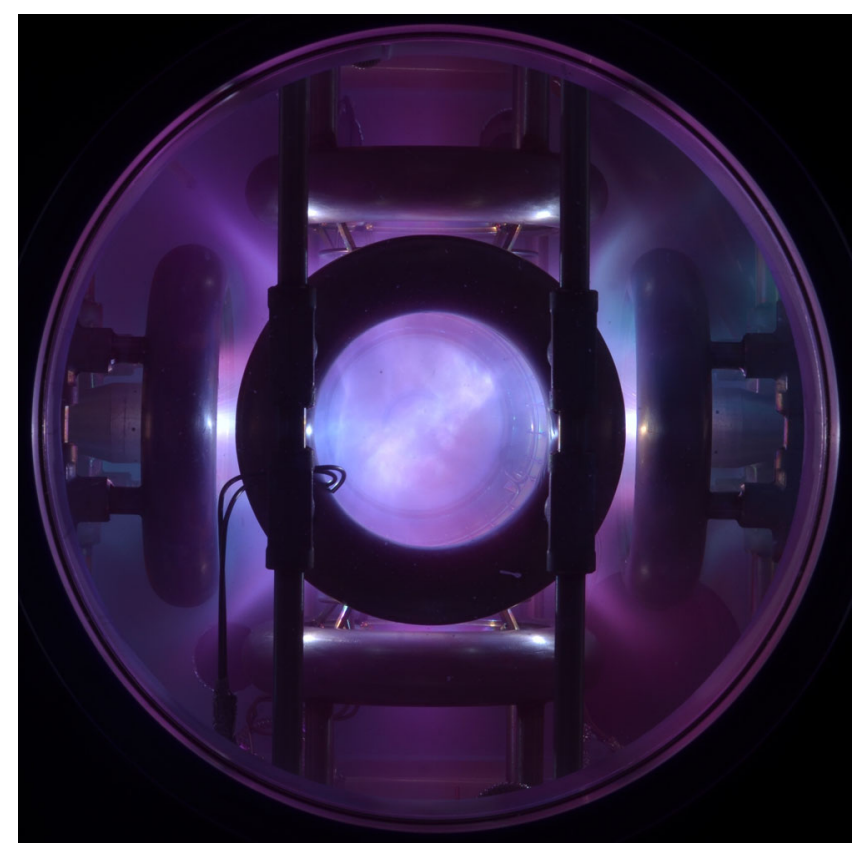

FIG. 3. Time-integrated raw visible light image of plasma from a high- $\beta$ shot (no. 15 640).

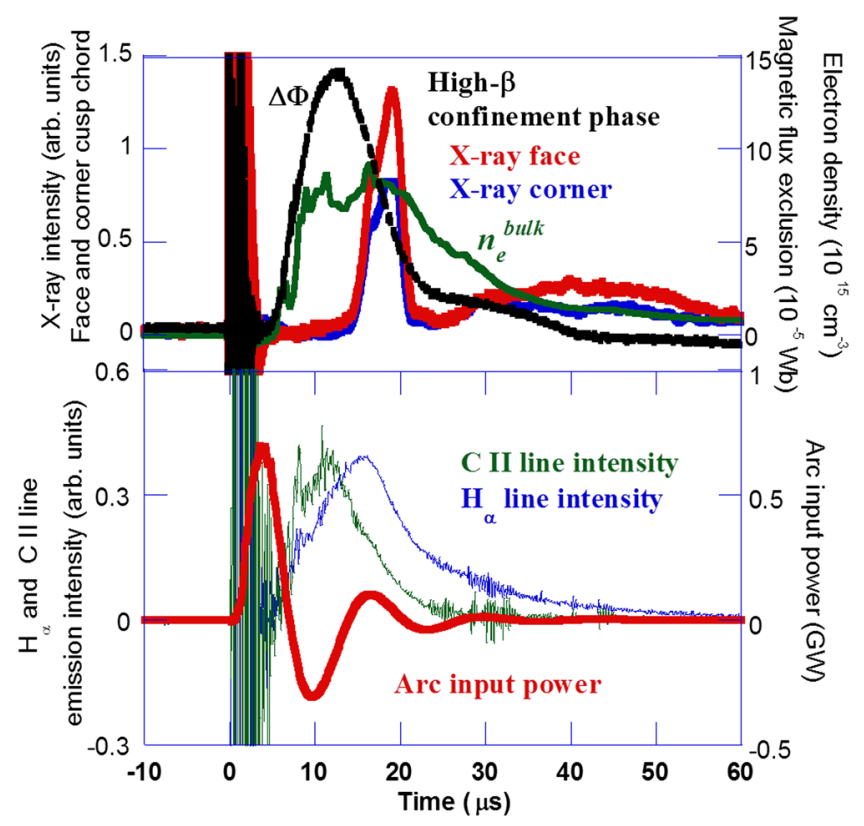

FIG. 4. Time-resolved experimental results showing enhanced high-energy electron confinement of high- $\beta$ plasma state in a magnetic cusp configuration.

the electron beam is turned on $30 \mu$ s before gun plasma injection and operates with $\sim 3 \mathrm{~A}$ of injection current at $7.2 \mathrm{kV}$ until $t=150 \mu \mathrm{s}$. At $t=0$, two coaxial plasma injectors are switched on with $700 \mathrm{MW}$ combined peak input power for $\sim 7 \mu$ s. Despite ringing in the underdamped gun circuit, the plasma injection provides high-density plasma in the cusp system with a rapid rise and a gradual decay, as measured by a heterodyne laser interferometer. The interferometer results are consistent with the plasma density estimate by Stark broadening of the $H_{\alpha}$ line. Plasma density, marked $n_{e}^{\text {bulk }}$, increases to $9 \times 10^{15} \mathrm{~cm}^{-3}$ at $t=9 \mu$ s as the plasma from the injectors is successfully transported to the center of the magnetic cusp system. The plasma density stays nearly constant until $t=20 \mu$ s and decreases gradually to $5 \times 10^{15} \mathrm{~cm}^{-3}$ at $t \approx 25 \mu \mathrm{s}$ and $2.5 \times 10^{15} \mathrm{~cm}^{-3}$ at $t \approx 32 \mu$ s. Flux-exclusion signals from flux loops, marked as $\Delta \Phi$, show a clear sign of a diamagnetic effect from the high- $\beta$ plasma formation in the cusp magnetic fields. The flux loop data peak at $1.4 \times 10^{-4} \mathrm{~Wb}$ of flux exclusion at $t=12.7 \mu \mathrm{s}$ and decrease to $0.4 \times 10^{-4} \mathrm{~Wb}$ at $t \approx 21 \mu \mathrm{s}$. The excluded flux measurement of $1.4 \times 10^{-4} \mathrm{~Wb}$ is equivalent to $10 \%$ of the vacuum magnetic flux at the location of the flux loop. Flux exclusion indicates a sharpening of the magnetic boundary, though the detailed shape of the magnetic field is not measured in the current work. At high $\beta$, diamagnetic plasma currents produce a magnetic field that cancels the vacuum magnetic field inside the plasma and adds to it outside the plasma. This change in magnetic field is what the flux loop is measuring. Separately, visible photodiode data also indicate the injection of plasma and its gradual decay based on $H_{\alpha}$ and C II line emission intensity. The faster decrease in the C II line 
intensity indicates cooling of the injected plasma after initial injection.

In comparison to the signals discussed above, the hard$\mathrm{x}$-ray emission intensity shows very distinctive features in the time domain. Prior to plasma injection, the $\mathrm{x}$-ray diode signals between $t=-10 \mu \mathrm{s}$ and $t=0$ provide an estimate for the $\mathrm{x}$-ray background data since there are no plasma ions to produce bremsstrahlung during this time period. The low background signals in the $\mathrm{x}$-ray diodes demonstrate good spatial collimation of $\mathrm{x}$-ray detectors. Covering any metallic surfaces in the line of sight of the x-ray detectors with plastic materials keeps $K \alpha$ emission from these surfaces below the Kapton filter threshold energy of $2 \mathrm{keV}$. Initially, the x-ray signals remain low until $12 \mu \mathrm{s}$ after plasma injection although the plasma density reaches its peak value of $9 \times 10^{15} \mathrm{~cm}^{-3}$ at $t=9 \mu \mathrm{s}$. After the peaking of flux data at $12.7 \mu \mathrm{s}$, both $\mathrm{x}$-ray diodes register strong increases in hard-x-ray emission, while the bulk plasma density varies little. The increase in $\mathrm{x}$-ray emission builds up from $t=14.5 \mu \mathrm{s}$ to $t=19 \mu \mathrm{s}$, while all other plasma diagnostics indicate gradual decay of injected plasma in the cusp. At $t=19 \mu \mathrm{s}$, the $\mathrm{x}$-ray emission intensity from both viewing channels rapidly decreases toward zero within $1.5 \mu \mathrm{s}$. The plasma density shows only gradual decrease during that time period and there is no sudden change in any other diagnostic. We note that the fast oscilloscope range is set too low for the $\mathrm{x}$-ray signal viewing through the cusp corners of the coils, resulting in artificial saturation in that channel.

This temporal behavior of the $\mathrm{x}$-ray emission signals can be explained as follows, clearly demonstrating the relation between high- $\beta$ plasma in the cusp magnetic field and the improved electron beam confinement as postulated by Grad and others $[1,4,8]$. Initially, the beam electrons are confined poorly in the magnetic cusp system with a low- $\beta$ plasma, resulting in low beam density and low $\mathrm{x}$-ray emission. After the intense plasma injection, the cusp magnetic field is altered by the diamagnetism of the high- $\beta$ plasma. This altered configuration, similar to Fig. 1(b), gives enhanced electron confinement as predicted theoretically. The increase in hard-x-ray emission shows that beam electrons are now better confined in the magnetic cusp in the presence of high- $\beta$ plasma and the beam electron density starts increasing in time. Since the beam current is limited to $3 \mathrm{~A}$, the increase in beam density and, therefore, the $\mathrm{x}$-ray emission intensity is gradual in time. The rise time of the $\mathrm{x}$-ray signal gives a preliminary estimate of the electron confinement time of $\sim 2.3 \mu \mathrm{s}$, half of the observed rise time. At $2.3 \mu \mathrm{s}$, the beam electrons are confined for $\sim 330$ bounces in the cusp at high $\beta$. In comparison, a previous study estimates a beam electron confinement time of $50 \mathrm{~ns}$ or $\sim 7$ bounces at low $\beta$ [14]. Determining the actual value of the confinement time, as opposed to the enhancement, is not possible due to the transient nature of the background plasma that produces the high- $\beta$ state. The present experimental setup does not have a subsequent plasma heating system after the initial plasma injection. Therefore, the high- $\beta$ plasma cannot be maintained indefinitely against plasma cooling and eventual loss of injected electron density. The enhanced plasma confinement phase is only temporary and it soon reverts back to the poor confinement phase as plasma $\beta$ decreases. When this transition occurs, all the previously confined high-energy electrons rapidly leave the magnetic cusp, causing the observed rapid decrease in x-ray emission at $t=21 \mu \mathrm{s}$. Electron-beam injection of sufficient power might prolong the duration of a good plasma confinement regime, but in the present experiment, the beam is far too weak to achieve this.

This temporal relationship between the x-ray signal and the inferred plasma $\beta$ by excluded flux measurement demonstrates a clear effect of high- $\beta$ plasma for enhanced confinement of high-energy electrons in the cusp. On the other hand, the x-ray data also show that this enhanced confinement persists for an extended duration while $\beta$ in the cusp decreases significantly. This persistence of enhanced confinement is surprising and we do not understand it at this point. While the transition to enhanced confinement at high $\beta$ had been theoretically predicted by Grad and others, we are not aware of any previous work on transition from enhanced confinement to poor confinement with decreasing $\beta$ in the cusp. Further study will be necessary to understand the transition between low $-\beta$ and high- $\beta$ states in the cusp and its effects on plasma confinement.

The key advance of the current work in validating the high- $\beta$ plasma cusp confinement stems from the use of an electron beam and beam-induced hard-x-ray measurement to measure the confinement property. This is because an electron beam at $7 \mathrm{keV}$ has a transit time of $7 \mathrm{~ns}$ to move across the cusp system, compared to several microseconds during which the high $\beta$ state is maintained. This provides the temporal resolution necessary to measure the change in confinement of the beam electrons by the magnetic cusp system during the plasma injection. This feature was absent in all previous magnetic cusp experiments. Additionally, the beam electrons have minimal coupling with the dense background plasma due to their high energy and low density, which avoids diagnostic complexities in separating losses of ions and electrons. High-energy electron confinement is a critical issue in the Polywell fusion concept, which motivated the use of an electron beam as a diagnostic to measure the cusp confinement.

Figures 5 and 6 show variation in $\mathrm{x}$-ray emission characteristics as plasma injection power and cusp magnetic flux density are varied. The plots are generated using the $\mathrm{x}$-ray signals from viewing the cusp via the centers of coils; the characteristics of $\mathrm{x}$-ray signals from the corners of cusps are qualitatively identical. In Fig. 5, the plasma injection power is varied from 220 to $700 \mathrm{MW}$ for a constant coil current generating $2.7 \mathrm{kG}$ of magnetic field at the cusp points near the plasma injectors. The x-ray signal results show that improvement in electron-beam confinement occurs only 

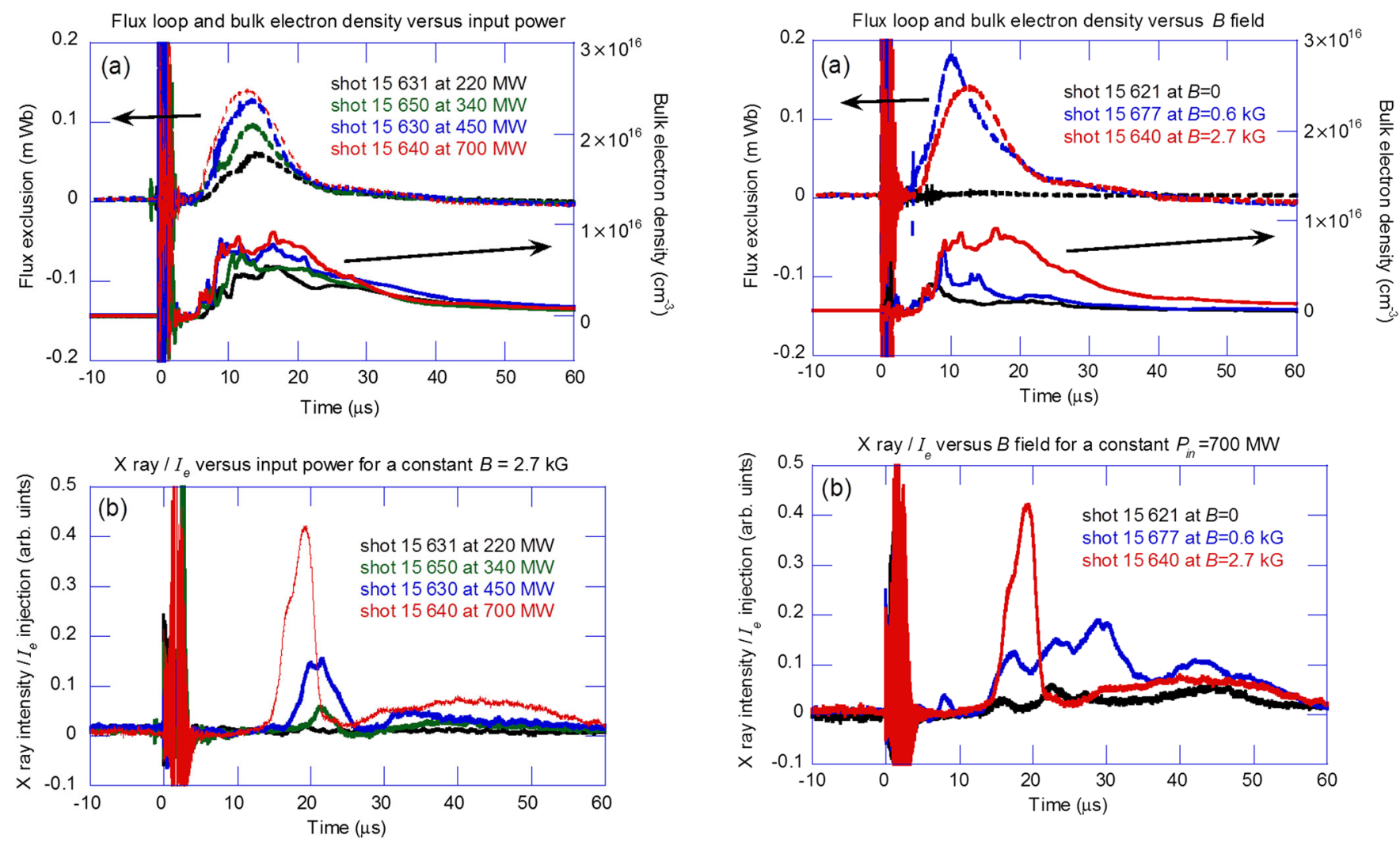

FIG. 5. Plasma parameters as a function of plasma injection power at $B$ field of $2.7 \mathrm{kG}$ : (a) flux exclusion and bulk electron density, (b) normalized x-ray emission intensity.

when there is a sufficient input power or energy in the plasma injection. In comparison, the excluded flux and bulk plasma density increase with plasma injection power. Figure 6 shows the $\mathrm{x}$-ray emission as a function of cusp magnetic field for a constant plasma injection power at $700 \mathrm{MW}$. At $B=0$, there is no diamagnetism and the $\mathrm{X}$-ray signal shows no distinctive structure, despite a clear indication of plasma injection from the density measurement albeit at a low level. Weak $x$-ray emission with slow and gradual rises and decays is explained by $\mathrm{x}$-ray bremsstrahlung from high- $Z$ impurities, such as tungsten from the coaxial gun electrodes. This has been verified by timeresolved visible emission spectroscopy using a gated CCD measuring various tungsten impurity line intensities compared to $\mathrm{C}$ line intensities. At $B=0.6 \mathrm{kG}$, the $\mathrm{x}$-ray result shows changes in electron-beam confinement, but the signal is complex to interpret. In comparison, at $B=2.7 \mathrm{kG}$, the $\mathrm{x}$-ray result clearly shows a well-defined period of large increase in electron-beam confinement. The excluded magnetic flux shows faster peaking and bigger diamagnetic effects at $B=0.6 \mathrm{kG}$ compared to $B=2.7 \mathrm{kG}$. The peak flux exclusion for the $B=0.6 \mathrm{kG}$ case is equivalent to $44 \%$ of the vacuum magnetic flux, compared to $10 \%$ for $B=2.7 \mathrm{kG}$. The injected plasma density is comparable initially, while the density decays rather rapidly for $B=0.6 \mathrm{kG}$. Though the detailed analysis of these results

FIG. 6. Plasma parameters as a function of magnetic field at 700 MW injection power: (a) flux exclusion and bulk electron density, (b) normalized x-ray emission intensity.

is beyond the scope of this work, the results indicate that a level of balance between the magnetic field pressure and the injected plasma pressure may be a key to an improvement in electron-beam confinement.

\section{COMPUTATIONS AND SCALING IMPLICATIONS}

The theory of high- $\beta$ plasma injection and electron-beam confinement has also been investigated using the threedimensional particle-in-cell code iPIC3D. iPIC3D is a massively parallel code solving the Vlasov-Maxwell system of equations using an implicit time scheme and has been used extensively in simulating magnetic reconnection and space plasma weather [19]. Initial results from iPIC3D were promising in that high- $\beta$ plasma injection resulted in strong diamagnetic effects and a significant change in magnetic field in a cusp. At present, available computational resources limit the numerical study of confinement due to insufficient spatial resolution and number of particles required to reduce the noise level. As a result, observations of improved confinement are limited. Further discussion and results of these simulations are available in the Appendix.

The present experimental result is a major step toward a Polywell fusion reactor in that it validates the conjecture that high-energy electron confinement is improved in a 
high- $\beta$ plasma. However, two additional measurements are needed to estimate the performance of a Polywell fusion reactor. The first is to quantitatively determine the loss rate. The second is to measure the efficiency of ion acceleration by electron-beam injection. For the purpose of discussion, we estimate the power balance for a 1-m-radius hexahedral D-T Polywell fusion reactor operating at $\beta=1$ with a magnetic field of $7 \mathrm{~T}$ at the cusp points and an electronbeam injection energy at $60 \mathrm{kV}$. This calculation is based on two assumptions: (1) the electron loss rate in Eq. (1) is correct and (2) the efficiency of ion acceleration via a potential well can be made good enough to convert $50 \%$ of electron-beam injection energy into an average ion energy. From Eq. (1), the electron loss current is $254 \mathrm{~A}$ per cusp for the electron density $2 \times 10^{15} \mathrm{~cm}^{-3}$ and electron energy at $60 \mathrm{keV}$ at the cusp points. Since there are 14 cusps in the hexahedral system, the required electron-beam power to maintain a $\beta=1$ state would be 213 MW. Separately, this system will lose an additional $51 \mathrm{MW}$ of power via bremsstrahlung radiation for an average electron temperature of $60 \mathrm{keV}$, assuming no ions other than hydrogen isotopes are present [20]. In comparison, the expected D-T fusion power output would be $1.9 \mathrm{GW}$ for a D-T cross section of $1.38 \mathrm{~b}$ at a center-of-mass energy of $30 \mathrm{keV}$ [21]. Though speculative at this point, this simple power balance scaling, coupled with the observed good plasma stability of a magnetic cusp system, indicates that the Polywell may emerge as an attractive concept for a compact fusion power reactor.

\section{CONCLUSIONS}

The present experimental results demonstrate for the first time that high- $\beta$ plasma operation can dramatically improve high-energy electron confinement in the magnetic cusp system. The experimental results also show that the enhanced confinement persists even after $\beta$ decreases significantly in the cusp, requiring further investigation. This enhanced high-energy electron confinement resolves the key issue of poor electron-beam confinement in the low- $\beta$ cusp system that had impeded the progress of the Polywell fusion concept to date. The current plan is to extend the present work with increased electron beam power to sustain the high- $\beta$ plasma state and to form an electrostatic well. If the deep potential well can be formed and the scaling of the electron-beam confinement is found to be favorable, as conjectured by Grad and others, it may be possible to construct a compact high- $\beta$ fusion power reactor based on the Polywell concept.

\section{ACKNOWLEDGMENTS}

We would like to thank John Santarius at University of Wisconsin for his theoretical support and discussions, Malcolm Fowler at McFarland Instrumentation for his assistance in x-ray measurements, Greg Dale and Robert Aragonez at Los Alamos National Laboratory (LANL) for their assistance in pulsed power system construction, Glen Wurden at LANL for his assistance in design and operation of visible spectroscopy and x-ray diagnostics, Kim Gunther and Marc Curtis at Heatwave and Ken Williams at Applied Science and Engineering for their assistance in development of the electron-beam injector system, Elizabeth Merritt and Mark Gilmore at University of New Mexico for their assistance in development of the laser interferometer, and Mike Wray, Noli Casama, and Kevin Wray at EMC2 for their laboratory operation support. This work was performed under Contract No. N68936-09-0125 awarded by the U.S. Department of Defense.

\section{APPENDIX: ADDITIONAL INFORMATION}

\section{1. iPIC3D numerical simulation}

We attempt to numerically simulate the physics discussed in this paper. Since the physics of diamagnetism is not related to the Debye length scale, we choose the implicit method [22] that relaxes the length and time scale requirement of the simulation, compared to the explicit particle-in-cell model. The present simulation resolves the electron cyclotron time scale and the electron skin depth, instead of the electron plasma frequency and the Debye length. The simulation utilizes an electron mass of $1 / 256$ of the hydrogen ion mass. The simulation utilizes the same hexahedral geometry of the experiments with the following changes. The total simulation volume is a cube with $38 \mathrm{~cm}$ edge length (or $54,872 \mathrm{~cm}^{3}$ ) and a conducting metal boundary corresponding to the vacuum chamber. The coil radius is $6.4 \mathrm{~cm}$ and the linear distance between two opposing coils is $20.4 \mathrm{~cm}$. The vacuum $B$-field value is $1.4 \mathrm{~T}$ at the cusps near the center of the coils. These parameters represent a scaling of the experimental apparatus that was tractable with available computational resources. Uniform density plasma is initially loaded in a cube of $7.3 \mathrm{~cm}$, centered in the simulation. The subsequent evolution is followed solving the full set of Maxwell equations for the fields and classical Newton equations for the particles. We report two cases, one with initial density equal to $2 \times 10^{15} \mathrm{~cm}^{-3}$, and the other with $2 \times 10^{14} \mathrm{~cm}^{-3}$. An initial electron temperature is set at $135 \mathrm{keV}$ and the ion temperature is set at $34 \mathrm{keV}$ for both cases. We use a grid of $160^{3}$ cells arranged in a topology of $10 \times 10 \times 10$ processors. At its peak, the simulation has about $1.6 \times 10^{6}$ particles, the number on average decreasing as the plasma decays. The simulations are run with the iPIC3D code [19]. We present two movies documenting the diamagnetic effect with frames taken at every 2500 cycles or $1.27 \times 10^{-8} \mathrm{~s}$. Movie 1 (M1) in the Supplemental Material [23] shows the high initial density case with the density shown in volume representation in color, superimposed to a representation of selected surfaces of constant magnitude of the magnetic field, while movie 2 (M2) shows the low initial density case. rho $1=1$ corresponds 
to the plasma density of $1 \times 10^{16} \mathrm{~cm}^{-3}$ and $B \mathrm{Mag}=1$ corresponds to the magnetic field of $1.38 \times 10^{3} \mathrm{~T}$.

As time evolves, the initial plasma expands and pushes the magnetic field out, providing clear evidence of the diamagnetic effect and of the formation of steeper gradient at the boundary with a more localized drop of the magnetic field compared with its initial shape. While the steep gradients are not fully resolved at the boundary, we have conducted a grid and time step convergence study that confirms that the particle loss and energy conservation are fully converged at the level of resolution used. The case with lower initial density shows a weak diamagnetic response, while the higher initial density case shows a strong response. The surface of $\beta=1$ has a particular importance in determining the diamagnetic effect, because there the plasma and magnetic pressure are nearly equal. This surface expands as the initial plasma loaded centrally expands and relaxes towards equilibrium with the magnetic field. We report such expansion in the third movie provided in the Supplemental Material [23], showing the expanding surface $\beta=1$ for the high-density case in movie 3 (M3). Finally, we compare the precise geometry of the $\beta=1$ surface for two cases at the cycle 10000 , corresponding to time $t=5.08 \times 10^{-8} \mathrm{~s}$ in movie 4 (M4) and movie 5 (M5) [23]. The movie rotates the view to allow the viewer to appreciate the 3D structure.

\section{Time-resolved measurements of tungsten impurities}

After more than 200 plasma injections, the tungsten cathode in the plasma coaxial gun shows significant erosion, as shown in Fig. 7. Since tungsten is a strong x-raygenerating target material when exposed to the electron beam, injection of tungsten impurities into the magnetic cusp has been investigated in the visible spectral range from 390 to $450 \mathrm{~nm}$ with the use of a spectrometer coupled with a gated CCD camera. Figures 8 and 9 show $8-\mu$ s timeintegrated spectra from the cusp plasma with the same gain setting. Figure 8 shows the plasma emission spectrum during the initial high $\beta$ state, between 12 and $20 \mu$ s after the plasma injection. Figure 9 shows the plasma emission spectrum after the plasma decay, between 42 and $50 \mu \mathrm{s}$ after the plasma injection. During the initial high- $\beta$ state, the emission spectrum is dominated by carbon lines with contributions from tungsten ion lines. In comparison, the emission spectrum is dominated by tungsten neutral lines after the plasma decay. Figure 10 shows relative brightness of carbon and tungsten lines as a function of time. The signals are normalized to their brightness at $12 \mu \mathrm{s}$ after the plasma injection. In addition, signals from photodiodes, filtered to look at hydrogen and ionized carbon $\left(H_{\alpha}\right.$ and C II), are plotted for comparison. The results indicate that tungsten impurities are introduced to the cusp plasma region gradually and on a slower time scale than the high- $\beta$ plasma injection. This is consistent with weak x-ray emission in the experiment with slow and gradual rises and decays.

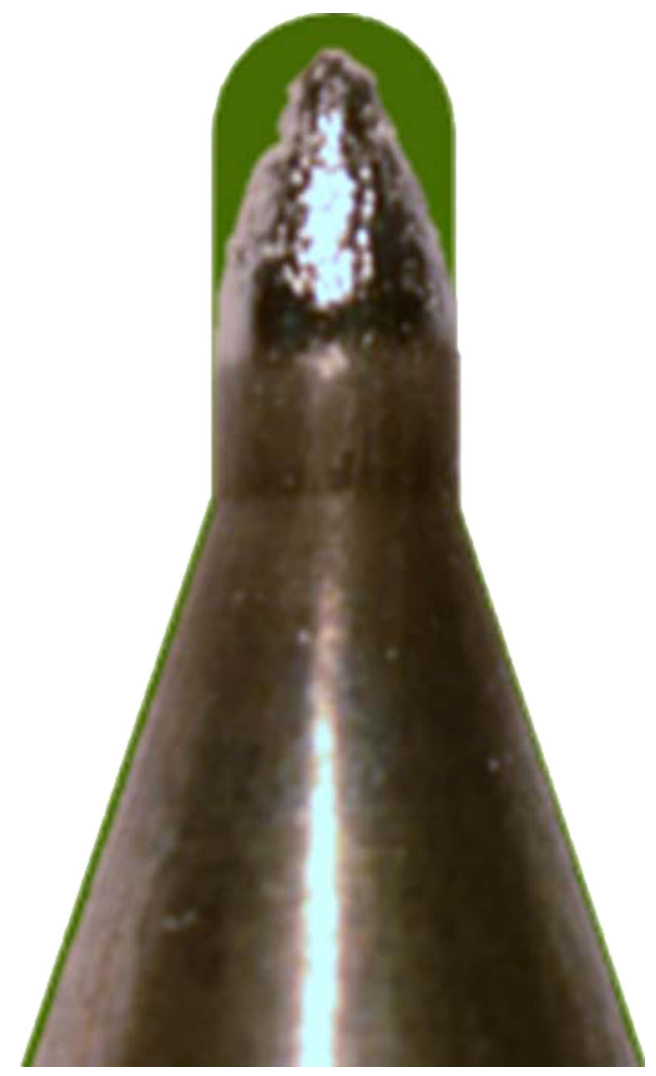

FIG. 7. A close-up photograph of a tungsten cathode after about 200 plasma injections. The width of the central tip is $2 \mathrm{~mm}$ and the green area highlights material erosion.

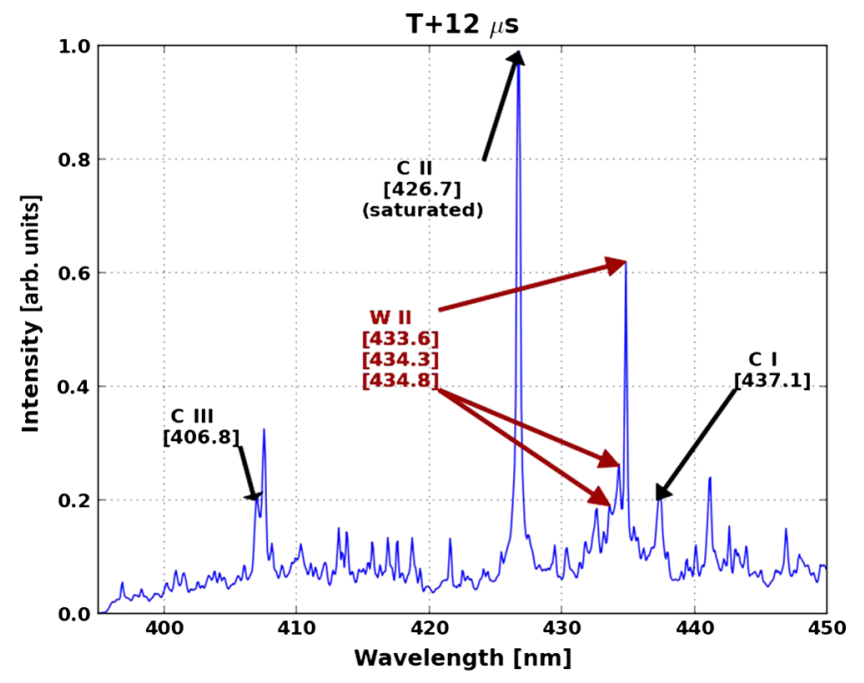

FIG. 8. Time-integrated spectra of the cusp plasma between 12 and $20 \mu \mathrm{s}$ after the plasma injection.

\section{Additional shot data showing enhanced high-energy electron confinement}

As pointed out in this paper, the use of a high-energy electron beam provides a powerful diagnostic tool to investigate the time evolution of cusp confinement. It is 


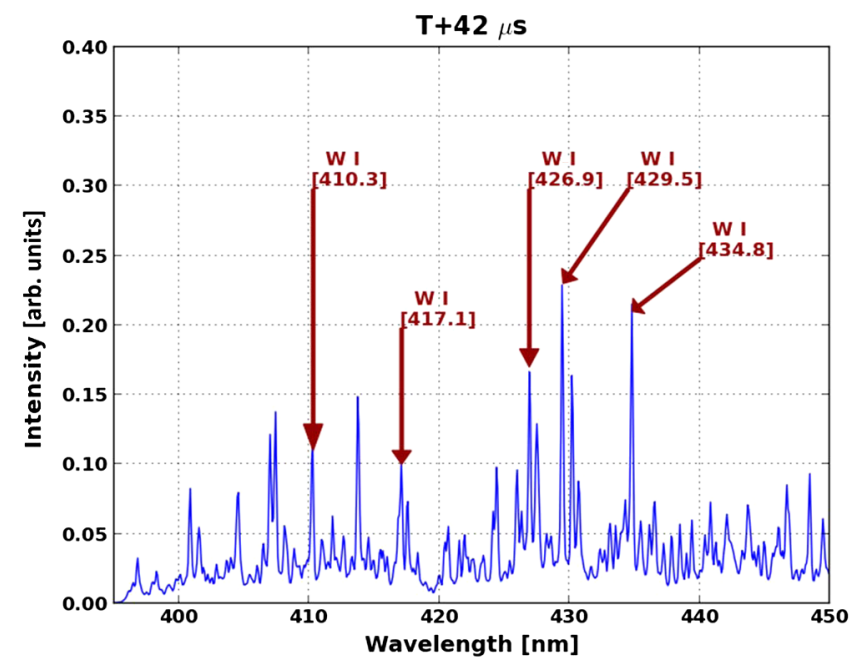

FIG. 9. Time-integrated spectra of the cusp plasma between 42 and $50 \mu \mathrm{s}$ after the plasma injection.

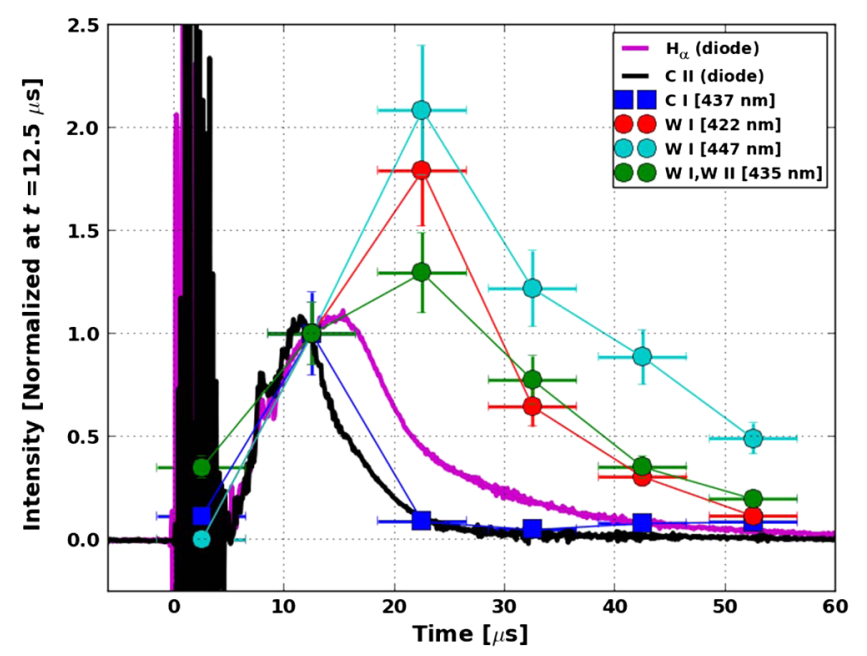

FIG. 10. Temporal variation of plasma line emission intensities, from ensemble of six plasma shots. The magenta lines and black lines are the average filtered photodiode signals for hydrogen $\alpha$ and carbon ion lines. It is noted that the diode signals have less than a $10 \%$ variation from shot to shot.

noted, however, that the plasma injection method used in the present work using high-power solid arcs results in significant variation in the observed $x$-ray signals. Figure 11 shows the $\mathrm{x}$-ray and excluded flux results for several discharges with 700-MW input power and $2.7 \mathrm{kG}$ cusp $B$ field. While there are substantial fluctuations in both $\mathrm{x}$-ray intensity and flux loop signals, several temporal features are consistently reproducible and, thus, discussed in the main text: (1) enhanced confinement of high-energy electrons (from the inflection point in X-ray intensity) begins $1-2 \mu$ s after the peak of flux exclusion, (2) the rise in x-ray intensity is gradual in time, followed by a rapid decrease after several $\mu \mathrm{s}$, and (3) enhanced confinement of high-energy electrons persists even when the flux exclusion (or inferred $\beta$ in the

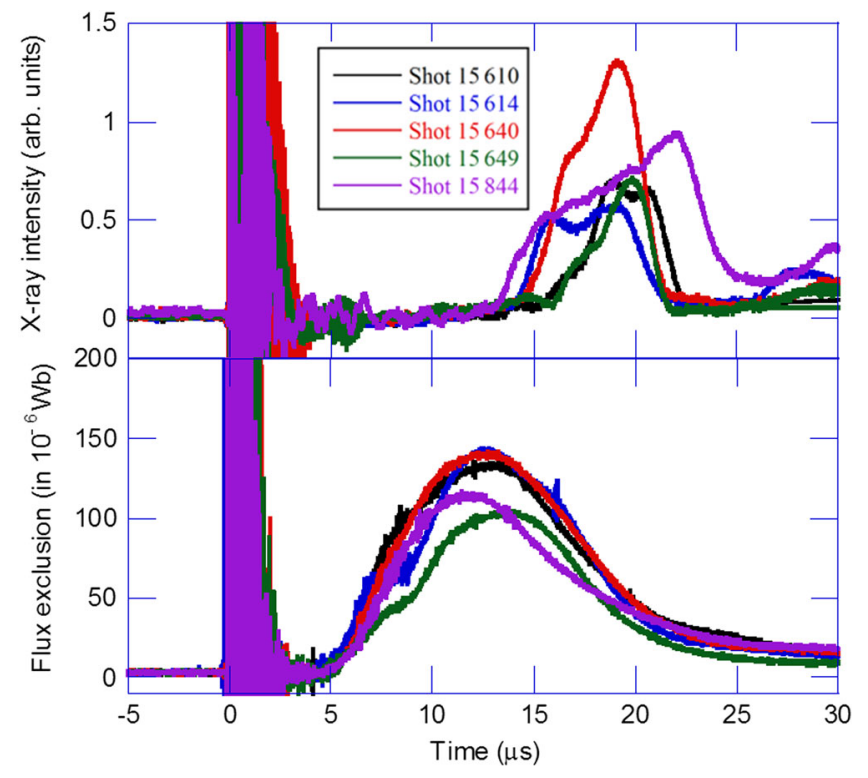

FIG. 11. X-ray emission intensity and flux exclusion from 5 high$\beta$ shots with 700-MW input power and 2.7-kG cusp $B$ field (shots no. 15610 , no. 15614 , no. 15640 , no. 15649 , and no. 15844 ).

cusp) decreases significantly. Two experimental improvements will be of great interest for future investigation of transition between poor confinement at low $\beta$ and enhanced confinement at high $\beta$ : (1) a more reproducible and controllable plasma start-up method to form high- $\beta$ plasma in the cusp and (2) a multitude of $B$-dot probes to spatially and temporally resolve the magnetic field profile.

[1] A. S. Bishop, Project Sherwood: The U. S. Program in Controlled Fusion (Addison-Wesley, Reading, MA, 1958).

[2] J.L. Tuck, A new plasma confinement geometry, Nature (London) 187, 863 (1960).

[3] J. Wesson, Tokamaks (Clarendon Press, Oxford, 1997).

[4] H. Grad, in Proceedings of the Conference on Thermonuclear Reactions (U.S. Atomic Energy Commission, Washington, DC, 1955), p. 115.

[5] I. Spalding, in Advances in Plasma Physics, edited by A. Simon and W. B. Thompson (Wiley, New York, 1971).

[6] M. G. Haines, Plasma containment in cusp-shaped magnetic fields, Nucl. Fusion 17, 811 (1977).

[7] ITER Physics Basis Editor et al., Iter physics basis, Nucl. Fusion 39, 2137 (1999).

[8] J. Berkowitz, K. O. Friedrichs, H. Goertzel, H. Grad, J. Killeen, and E. Rubin, in Proceedings of the Second U.N. International Conference on Peaceful Uses of Atomic Energy, Geneva, 1958 (United Nations, Geneva, 1958), Vol. 31, p. 171.

[9] N. A. Krall, M. Coleman, K. C. Maffei, J. A. Lovberg, R. A. Jacobsen, and R. W. Bussard, Forming and maintaining a potential well in a quasispherical magnetic trap, Phys. Plasmas 2, 146 (1995).

[10] J. Marshall, Jr., U. S. Patent No. 2,961,559 (1960). 
[11] R. E. Pechacek, J. R. Greig, M. Raleigh, D. W. Koopman, and A. W. DeSilva, Measurement of the Plasma Width in a Ring Cusp, Phys. Rev. Lett. 45, 256 (1980).

[12] K. N. Leung, N. Hershkowitz, and K. R. MacKenzie, Plasma confinement by localized cusps, Phys. Fluids 19, 1045 (1976).

[13] R. W. Bussard, Some physics considerations of magnetic inertial-electrostatic confinement: A new concept for spherical converging-flow fusion, Fusion Sci. Technol. 19, 273 (1991).

[14] N. A. Krall, The polywell: A spherically convergent ion focus concept, Fusion Sci. Technol. 22, 42 (1992).

[15] R. W. Bussard, in Proceedings of the 57th International Astronautical Congress, 2006 (International Astronautical Federation, Valencia, Spain, 2006), p. 8105.

[16] P. T. Farnsworth, U. S. Patent No. 3,386,883 (1968).

[17] R. L. Hirsch, Inertial electrostatic confinement of ionized fusion gases, J. Appl. Phys. 38, 4522 (1967).
[18] W. C. Elmore, J. L. Tuck, and K. M. Watson, On the inertial-electrostatic confinement of a plasma, Phys. Fluids 2, 239 (1959).

[19] S. Markidis, G. Lapenta, and Rizwan-uddin, Multi-scale simulations of plasma with iPIC3D, Math. Comput. Simul. 80, 1509 (2010).

[20] J. D. Huba, Plasma Formulary (Naval Research Laboratory, Washington, DC, 2013), http://www.nrl.navy.mil/ppd/ content/nrl-plasma-formulary.

[21] H. S. Bosch and G. M. Hale, Improved formulas for fusion cross-sections and thermal reactivities, Nucl. Fusion 32, 611 (1992).

[22] J. U. Brackbill and D. W. Forslund, An implicit method for electromagnetic plasma simulation in two dimensions, J. Comput. Phys. 46, 271 (1982).

[23] See Supplemental Material at http://link.aps.org/ supplemental/10.1103/PhysRevX.5.021024 for movies of $3 \mathrm{D}$ simulation results as described in the Appendix. 